\title{
Idade de maior sensibilidade de tilápias-do-nilo aos tratamentos de masculinização por banhos de imersão
}

\section{Robie Allan Bombardelli1, Eduardo Antônio Sanches², Donovan Filipe Henrique Pinto², Ronan Maciel Marcos ${ }^{2}$, Leandro Barbero ${ }^{3}$}

\footnotetext{
1 Curso de Engenharia de Pesca da Universidade Estadual do Oeste do Paraná - UNIOESTE - Campus de Toledo. Rua da Faculdade, no 645 - Jardim La Salle - CEP: 85903-000, Toledo/PR.

2 Graduando em Engenharia de Pesca da Universidade Estadual do Oeste do Paraná.

${ }^{3}$ Mestrando do Programa de Pós-Graduação em Zootecnia da Universidade Estadual de Maringá.
}

RESUMO - Objetivou-se determinar o período ontogênico de maior sensibilidade de tilápias-do-nilo (Oreochromis niloticus) aos tratamentos de masculinização com $17 \alpha$-metiltestosterona (MT), realizados via banhos de imersão com 2,0 mg MT.L $\mathrm{L}^{-1}$ por um período de 36 horas. Nos tratamentos hormonais, foram utilizadas 3.000 tilápias distribuídas em 15 recipientes plásticos com capacidade para 2,0 L (unidades experimentais), em um delineamento experimental inteiramente casualizado, com cinco tratamentos e três repetições. Os tratamentos consistiram de um tratamento controle (tilápias de 16 DPE [dias pós-eclosão] submetidas à imersão sem hormônio) e da imersão dos peixes em idades correspondentes a 16, 20, 24 e 28 DPE. Os parâmetros avaliados foram peso (PF) e comprimento finais (CF), taxa de sobrevivência (S) e proporção sexual. Os resultados de masculinização evidenciaram relação quadrática entre a idade e as taxas de masculinização, de modo que os melhores resultados foram obtidos aos 23,30 DPE (73,02\% de machos). Os tratamentos não influenciaram as médias de PF, CF e S. A idade mais sensível aos tratamentos de masculinização via banhos de imersão para a linhagem de tilápia utilizada foi de 23,30 DPE.

Palavras-chave: andrógeno, ciclídeos, peixes, período ontogênico, reversão sexual, solução hormonal

\section{Most sensitivity age to masculinization of Nile tilapia by immersion baths}

\begin{abstract}
The present experiment was carried out to determine the different stages during ontogenesis of greater sensitivity to hormonal treatments of sex reversal of Nile tilapia larvae (Oreochromis niloticus) with $17 \alpha$-metiltestosterone (MT) by bath immersion in $2.0 \mathrm{mg}$ of $\mathrm{MT} . \mathrm{L}^{-1}$ for 36-h period. In the hormonal treatment, 3,000 Nile tilapia were allotted to $152 \mathrm{~L}$ - plastic containers (experimental units) as a complete randomized design, with five treatments and three replicates. The treatments consisted of the fish immersion at different ages: control (fishes immersed at 16 DAH [days after hatching] and without hormone), and 16, 20, 24, and 28 DAH. Final weight (FW), final length (FL), survival rate (SR) and sexual rate were the evaluated parameters. The masculinization results showed positive quadratic relationship between tilapia age and hormonal treatments, and this fact suggests that the best results were obtained at 23.30 DAH (73.02\% of males). No treatment effect on FW, FL and SR was observed. The most sensible age to the masculinization of Nile tilapia by immersion baths was of $23.30 \mathrm{DAH}$.
\end{abstract}

Key Words: androgen, chiclide, fish, hormonal solution, ontogenetic period, sex reversal

\section{Introdução}

Entre as espécies de peixes utilizadas na aqüicultura, as tilápias, especialmente do gênero Oreochromis, são algumas das mais promissoras (Stickney, 2000) em países de clima tropical ou subtropical (Campos-Ramos et al., 2003; Desprez et al., 2003). À exceção das carpas, as tilápias são os peixes mais cultivados no mundo (Alceste \& Jorry, 1998) e no Brasil (Borghetti et al., 2003). Tilápias do gênero Oreochromis, em especial Oreochromis niloticus, têm sido consideradas importantes para as condições de cultivo no Brasil por sua rápida taxa de crescimento, pela adaptabilidade aos diversos sistemas de cultivo e pela alta aceitação no mercado consumidor (Meurer et al., 2000; Boscolo et al., 2001).

Contudo, esses peixes apresentam desovas parceladas e baixa fecundidade (Phelps \& Popma, 2000), característica compensada pelas desovas assincrônicas, associada às altas taxas de sobrevivência das proles. A alta sobrevivência está relacionada a características específicas, como a 
incubação bucal dos ovos e/ou das larvas, o tamanho das larvas ao nascer e o cuidado parental (Turner \& Robinson, 2000).

Além dessas características reprodutivas, as tilápias apresentam ainda maturação sexual precoce, que pode acarretar problemas em condições de cultivo, como desvio da energia destinada ao crescimento para a reprodução, superpopulação e queda da qualidade de água (Stickney, 2000).

Na tentativa de minimizar os problemas relacionados à precocidade reprodutiva e à elevada prolificidade das tilápias em condição de cultivo (Stickney, 2000), diversos pesquisadores têm estudado técnicas visando à produção de populações monossexuais (Phelps \& Popma, 2000), como a incorporação de andrógeno na ração(Beardmore et al., 2001; Leonhardt, 1997; MacIntosh \& Little, 1995), queéa mais utilizada atualmente na tilapicultura. Apesar da simplicidade e facilidade de aplicação, esta técnica pode ocasionar reduzidas taxas de masculinização, como resultado de fatores (ambientais e hierarquias sociais) que influenciam a taxa de obtenção de ração (MacIntosh \& Little, 1995).

Neste sentido, a técnica de banhos de imersão, que consiste em exposições periódicas ou contínuas dos animais em solução contendo agente alterador sexo (Pandian \& Sheela, 1995), pode ser um processo alternativo, principalmente por minimizar o efeito de algumas características potencialmente influenciadas pelo método de incorporação de hormônios na ração (Gale et al., 1999; Beardmore et al., 2001) e por ser ambientalmente mais segura, pois possibilita o armazenamento do resíduo para posterior degradação (Gale et al., 1999) ou filtragem em carbono ativado (Specker \& Chandlee, 2003).

Mesmo que desde 1965 tenham sido realizados estudos envolvendo banhos de imersão em tilápias (Eckstein \& Spira, 1965; Torrans et al., 1988; Gale et al., 1999; Wassermann et al., 2000; Bombardelli et al., 2003; Wassermann \& Afonso, 2003; Hayashi et al., 2004; Bombardelli \& Hayashi, 2005a,b), esta técnica não é empregada comercialmente, pois não existe um protocolo efetivo.

Como as respostas aos tratamento hormonais são diferenciadas durante os diferentes estádios ontogênicos (Hiott \& Phelps, 1993; Koger et al., 2000; Krisfalusi \& Nager, 2000; Blázquez et al., 2001), o primeiro passo no estabelecimento de regimes efetivos de tratamento hormonal para reversão sexual deve ser a determinação do período de desenvolvimento ontogênico de maior sensibilidade dos animais aos tratamentos com hormônios exógenos (Blázquez et al., 1995; Piferrer, 2001). Em seguida, outros aspectos como duração dos tratamentos e dose de hormônios poderão ser ajustados (Strussmann et al., 1996).
Este trabalho foi realizado com o objetivo de determinar a fase ontogênica de maior sensibilidade de tilápias-do-nilo aos tratamentos com $17 \alpha$-metiltestosterona, via banhos de imersão, e seus efeitos sobre a reversão sexual e o desempenho zootécnico dos peixes.

\section{Material e Métodos}

O experimento foi realizado no Centro de Pesquisa em Aqüicultura Ambiental - CPAA/IAP e no Laboratório de Tecnologia da Reprodução Aplicada aos Animais Aquáticos Cultiváveis da Universidade Estadual do Oeste do Paraná - UNIOESTE - Toledo-PR. O experimento teve 55 dias de duração e foi realizado em duas fases, a primeira constituída pelos tratamentos hormonais e a segunda pela fase de crescimento dos animais até atingirem o comprimento mínimo necessário para avaliação dos resultados.

Foram utilizadas 4.000 tilápias-do-nilo (Oreochromis niloticus) provenientes de diversas proles de um mesmo grupo de reprodutores, obtidas por coleta manual dos ovos de fêmeas incubantes seguida de incubação artificial. As proles foram distribuídas em 20 tanques-rede (malha de $1,0 \mathrm{~mm}$ e 0,7 x 0,4 x 0,7 m de dimensão), em densidade de 200 tilápias por tanque. Os tanques-rede foram alojados inicialmente dentro de um viveiro escavado, revestido de alvenaria e com $16 \mathrm{~m}^{2}$ de área útil.

Na primeira fase, foram utilizados 3.000 peixes (Tabela 2) provenientes de 15 tanques-rede. Os peixes foram transferidos para 15 recipientes plásticos (unidades experimentais) com 2,0 L de volume útil, distribuídos em delineamento experimental inteiramente casualizado, com cinco tratamentos e três repetições, totalizando 15 unidades experimentais com 200 peixes. Os tratamentos foram organizados considerando os resultados prévios descritos por Bombardelli \& Hayashi (2005a) e consistiram da imersão das larvas em diferentes idades ou fases de desenvolvimento ontogênico: 16, 20, 24 e 28 dias pós-eclosão (DPE). Para comparação dos resultados, adotou-se um tratamento controle (16C), no qual animais de $16 \mathrm{DPE}$ foram submetidos ao mesmo procedimento de imersão, porém sem o uso de hormônio.

$\mathrm{O}$ andrógeno utilizado nos tratamentos foi o $17 \alpha$-metiltestosterona, em concentração de $2,0 \mathrm{mg}$ de $17 \alpha$-metiltestosterona. $L^{-1}$ de solução (Bombardelli \& Hayashi, 2005a) e o veículo de diluição, álcool etílico $\left(95^{\circ} \mathrm{GL}\right)$. Em todos os tratamentos, os banhos tiveram duração de 36 horas, período no qual as unidades experimentais foram mantidas sob aeração por pedra microporosa para possibilitar a constante homogeneização da solução (Gale et al., 1999) 
e evitar a hipóxia. A temperatura da água foi controlada e mantida entre 24,0 e $25,0^{\circ} \mathrm{C}$ e, ao fim dos tratamentos hormonais, foi armazenada para posterior tratamento e descarte.

Os outros cinco tanques-rede foram utilizados para a amostragem de 100 tilápias e determinação do peso e do comprimento inicial médio de cada tratamento.

Ao término de cada tratamento, os animais foram realojados em seus respectivos tanques-rede e, depois do último tratamento de imersão, foram transferidos desses tanques-rede para 15 viveiros escavados, revestidos em alvenaria (16 $\mathrm{m}^{2}$ de área útil), para manutenção da homogeneidade entre as densidades de estocagem. Na fase de crescimento, foram distribuídos em um delineamento inteiramente casualizado, com cinco tratamentos e três repetições, considerando unidade experimental cada viveiro escavado contendo 200 tilápias. Os viveiros foram mantidos sob renovação constante de água para compensar as perdas por infiltração e evaporação. Durante esta fase, os animais foram cultivados até atingirem o comprimento padrão mínimo de $2,5 \mathrm{~cm}$, necessário para a análise da taxa de masculinização. As larvas foram alimentadas à vontade, cinco vezes ao dia (Sanches \& Hayashi, 2001), com uma ração prática e sem hormônio (Tabela 1) formulada para atender às exigências nutricionais da espécie nesta fase de crescimento (38,6\% PB e $3.800 \mathrm{kcal} / \mathrm{kg}$ de ração), conforme descrito por Hayashi et al. (2002). Os alimentos utilizados na ração foram processados segundo técnica relatada por Hayashi et al. (1999) e Meurer et al. (2003a).

A temperatura da água dos viveiros foi medida diariamente por meio de termômetro de máxima e mínima, com precisão de $1^{\circ} \mathrm{C}$. Semanalmente, foram mensurados os demais parâmetros físico-químicos da água, como oxigênio dissolvido (mg.L $\left.\mathrm{L}^{-1}\right)$, condutividade elétrica $\left(\mu \mathrm{S} . \mathrm{cm}^{-1}\right)$ e $\mathrm{pH}$.

Ao final do experimento, os alevinos foram insensibilizados por choque térmico a aproximadamente $2{ }^{\circ} \mathrm{C}$, para determinação do peso $(\mathrm{g})$ e comprimento $(\mathrm{mm})$ final e da taxa de sobrevivência (\%). Em seguida, foram fixados inteiros em solução de formalina a $10 \%$ para análise sob microscopia óptica das gônadas coradas (Popma \& Green, 1990) e determinação da proporção entre os sexos.

Os dados obtidos foram submetidos à análise de variância a 5\% de probabilidade aplicando-se a análise de regressão e o teste Duncan para comparação múltipla de médias e avaliação do efeito dos tratamentos hormonais em comparação ao tratamento controle. O software utilizado nas análises estatísticas foi o Sistema de Análises Estatísticas e Genéticas - SAEG (UFV, 1997).
Tabela 1 - Composição percentual da ração experimental Table 1 - Ingredient composition of the experimental diet

\begin{tabular}{|c|c|}
\hline $\begin{array}{l}\text { Alimento } \\
\text { Food }\end{array}$ & $\begin{array}{c}\text { Quantidade (\%) } \\
\text { Amount }(\%)\end{array}$ \\
\hline $\begin{array}{l}\text { Farinha de vísceras de frango }{ }^{1} \\
\text { Poultry viscera meal }^{1}\end{array}$ & 54,63 \\
\hline $\begin{array}{l}\text { Farelo de soja } \\
\text { Soybean meal }\end{array}$ & 19,15 \\
\hline Levedura spray dried ${ }^{1}$ & 6,00 \\
\hline $\begin{array}{l}\text { Spray dried yeast } \\
\text { Milho }^{2}\end{array}$ & 7,68 \\
\hline Corn & \\
\hline $\begin{array}{l}\text { Óleo de } \text { soja }^{2} \\
\text { Soybean oil }\end{array}$ & 10,02 \\
\hline $\begin{array}{l}\text { Suplemento (min. + vit.) } \\
\text { Supplement }(\min +\text { vit })\end{array}$ & 2,00 \\
\hline $\begin{array}{l}\text { Sal } \\
\text { Salt }\end{array}$ & 0,50 \\
\hline $\begin{array}{l}\text { Antioxidante BHT } \\
\text { Antioxidant } B H T\end{array}$ & 0,02 \\
\hline
\end{tabular}

\section{Resultados e Discussão}

Os valores de temperatura $\left(24,45 \pm 2,31^{\circ} \mathrm{C}\right), \mathrm{pH}$ $(7,85 \pm 0,60)$, oxigênio dissolvido $\left(7,52 \pm 0,51 \mathrm{mg} . \mathrm{L}^{-1}\right)$ e condutividade elétrica $\left(0,16 \pm 0,05 \mu \mathrm{S} . \mathrm{cm}^{-1}\right)$ durante o período experimental não tiveram efeito $(\mathrm{P}>0,05)$ sobre os tratamentos e mantiveram-se nos limites considerados adequados para o cultivo de peixes (Boyd, 1990; SipaúbaTavares, 1995) e o bom desempenho da espécie (Popma \& Phelps, 1998).

Os resultados de desempenho zootécnico e proporção sexual das tilápias-do-nilo são apresentados na Tabela 2. A porcentagem de machos variou de 47,00 a 71,92\% e apresentou relação quadrática $(\mathrm{P}<0,05)$ entre a taxa de masculinização e a idade das larvas (Tabela 2), além de diferença $(\mathrm{P}<0,05)$ entre o tratamento controle e aqueles com hormônio (Tabela 2, Figura 1).

Os resultados de masculinização sugeriram a existência de um período de máxima sensibilidade aos tratamentos hormonais, aos 23,3 DPE (73,02\% de machos) para tilápiado-nilo (Oreochromis niloticus) (Figura 1).

A existência de um período ontogênico de maior sensibilidade aos tratamentos hormonais tem sido verificada em diversas espécies, como o salmão, Oncorhynchus kisutch (Goetz et al., 1979; Piferrer \& Donaldson, 1989), o sea bass, Dicentrarchus labrax (Blázquez et al., 1995), o medaka, Oryzias latipes (Koger et al., 2000) e a truta arco-íris, Oncorhynchus mykiss (Krisfalusi \& Nagler, 2000). 
Tabela 2 - Valores de desempenho zootécnico e proporção sexual de tilápias-do-nilo submetidas a banhos de imersão com 17 $\alpha$-metiltestosterona em diferentes períodos ontogênicos

Table 2 - Values of performance and sexual ratio of Nile tilapia (Oreochromis niloticus) submitted to bath immersion with $17 \alpha$-metiltestosterone at different stages during ontogenesis

\begin{tabular}{|c|c|c|c|c|c|c|}
\hline & \multicolumn{5}{|c|}{$\begin{array}{c}\text { Idade (DPE) } \\
\text { Age }(D A H)\end{array}$} & \multirow[t]{2}{*}{$\mathrm{CV}(\%)$} \\
\hline & $16 \mathrm{C}$ & 16 & 20 & 24 & 28 & \\
\hline Peso inicial (g) (Initial weight) & 0,011 & 0,011 & 0,021 & 0,044 & 0,101 & \\
\hline Peso final $(\mathrm{g})$ (Final weight) & 3,43 & 2,96 & 2,71 & 3,14 & 3,17 & $17,08^{\mathrm{ns}}$ \\
\hline Comprimento inicial $(\mathrm{mm})$ (Initial lenght) & 7,47 & 7,47 & 9,00 & 11,3 & 13,8 & \\
\hline Comprimento final $(\mathrm{mm})$ (Final lenght) & 40,70 & 40,56 & 40,27 & 41,70 & 42,60 & $6,81^{\mathrm{ns}}$ \\
\hline Sobrevivência (\%) (Survival) & 78,25 & 80,50 & 81,50 & 69,50 & 56,17 & $20,91^{\mathrm{ns}}$ \\
\hline Machos $(\%)^{1}$ (Male sex ratio) & $47,00^{\mathrm{b}}$ & $50,57^{\mathrm{b}}$ & $69,57^{\mathrm{b}}$ & $71,92^{\mathrm{a}}$ & $64,19^{\mathrm{b}}$ & $12,66^{*}$ \\
\hline Fêmeas $(\%)^{2}$ (Female sex ratio) & $53,00^{\mathrm{a}}$ & $10,47^{\mathrm{b}}$ & $1,99^{\mathrm{b}}$ & $4,52^{\mathrm{b}}$ & $9,55^{\mathrm{b}}$ & $67,19^{*}$ \\
\hline Intersexuais (\%) (Intersex sex ratio) & 0,00 & 38,96 & 28,44 & 23,56 & 26,26 & $25,71^{\mathrm{ns}}$ \\
\hline
\end{tabular}

$(P<0,05)$.

ns $(P>0,05)$.

$1 \hat{Y}=-154,415+19,553 x-0,420 x^{2} ; R^{2}=0,64, P=0,028$.

$2 \hat{Y}=105,880-9,417 x-0,214 x^{2} ; R^{2}=0,48, P=0,042$.

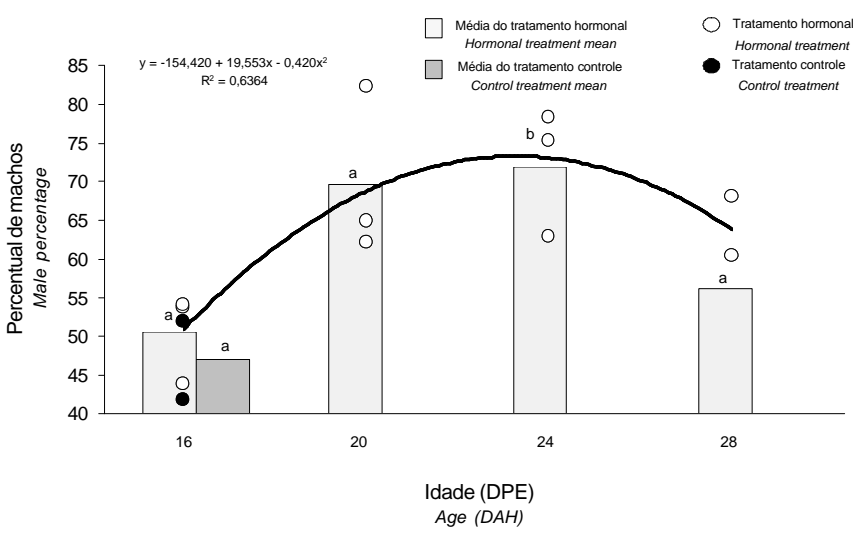

Figura 1 - Taxa de masculinização de tilápias-do-nilo submetidas a banhos de imersão com $17 \alpha$-metiltestosterona em diferentes períodos ontogênicos.

Figure 1 - Percentage of masculinization of Nile tilapia submitted to bath imersion with $17 \alpha$-metiltestosterone at different stages during ontogenesis.

Taxas de masculinização semelhantes às encontradas neste experimento foram obtidas por Gale et al. (1999), em larvas de tilápia-do-nilo submetidas a dois banhos de imersão consecutivos de 3 horas com $0,1 \mathrm{mg}$ de $17 \alpha$-metiltestosterona. $\mathrm{L}^{-1} \mathrm{em}$ dois períodos ontogênicos (10 e 13 dias após a fertilização). Esses autores obtiveram 73 e $83 \%$ de machos em dois ensaios consecutivos.

Apple \& Leboute (1995) realizaram banhos de imersão de 2 horas em tilápias-do-nilo com 7 e 14 DPE e verificaram que os tratamentos realizados no 140 DPE foram mais eficientes. Por outro lado, Wassermann et al. (2000) não verificaram consistência nos resultados de masculinização em tilápias-do-nilo com 14 DPE quando utilizaram banhos de imersão de 4 horas com $17 \alpha$-metiltestosterona. Fiest et al. (1995) verificaram a existência de um período de maior sensibilidade para truta arco-íris submetidas a tratamentos com $17 \alpha$-metiltestosterona. Contudo, observaram que a imersão resultou em vários graus de masculinização, enquanto a imersão associada à suplementação dietética foi $100 \%$ efetiva. Bombardelli \& Hayashi $(2005 a, b)$ também notaram efeito da idade sobre o sucesso na masculinização e na feminilização, respectivamente, de tilápias-do-nilo a partir de banhos de imersão que evidenciaram efeito linear positivo entre as taxas de masculinização e a idade dos animais até os 15 DPE, sugerindo que o período de maior sensibilidade aos tratamentos hormonais é superior a esta idade.

Wassermann \& Afonso (2003) registraram elevados índices de masculinização em tilápias-do-nilo $(91,60 \%$ de machos) submetidas a um único banho de imersão em 1,8 mg de $17 \alpha$-metiltestosterona. $\mathrm{L}^{-1}$ de solução aos $14 \mathrm{DPE}$. Esses resultados sugerem que o período apropriado ou de maior sensibilidade aos tratamentos antecede o término da diferenciação sexual morfológica, que, em tilápias-donilo ocorre por volta dos 33 DPE (Alvenida-Casauay \& Cariño, 1988).

Os resultados deste trabalho corroboram estas afirmações, pois o período de maior sensibilidade teórica foi aos 23,3 DPE, contudo, variações intra-específicas podem existir, pois os resultados variam em diferentes idades para a mesma espécie. Além disso, estes resultados indicam que a pressuposição de Yamamoto (1969) sobre a duração dos tratamentos hormonais durante todo o período de diferenciação sexual não sejam uma regra plena, o que pode ser comprovado pelos resultados obtidos por Piferrer \& Donaldson (1989), que, conhecendo e respeitando o período 
de maior sensibilidade aos tratamentos hormonais, obtiveram em Oncorhynchus kisutch grande eficiência nos tratamentos com $17 \alpha$-metiltestosterona e $17 \beta$-estradiol em banhos imersão de apenas 2 horas. O mesmo foi verificado por Gale et al. (1999), que realizaram dois banhos consecutivos (3 horas cada um) em tilápias-do-nilo (Oreochromis niloticus) utilizando $17 \alpha$-metildihidrotestosterona.

O peso final médio $(\mathrm{g})$, o comprimento final médio $(\mathrm{cm})$ e a taxa de sobrevivência (\%) não diferiram significativamente $(\mathrm{P}>0,05)$ entre os tratamentos (Tabela 2), o que está de acordo com os resultados obtidos por Koger et al. (2000), que verificaram que banhos de imersão em diferentes estádios de desenvolvimento não influenciaram significativamente a sobrevivência e o peso corporal de Oryzias latipes.

Apesar da inconsistência nos resultados de sobrevivência, deve-se observar que os tratamentos hormonais com larvas em idades mais avançadas resultaram, numericamente, em menores taxas de sobrevivência, o que, possivelmente, pode inviabilizar o processo. Bombardelli \& Hayashi (2005a) verificaram o mesmo comportamento para a sobrevivência, mas estas taxas para tilápias mais velhas permaneceram relativamente altas $(88,50 \%)$. Embora neste experimento tenha sido utilizada a mesma densidade de estocagem durante os banhos hormonais adotada por Bombardelli \& Hayashi (2005a) e Bombardelli \& Hayashi (2005b), a mortalidade pode estar relacionada ao tamanho dos peixes. Este fato elevou, conseqüentemente, a relação de estocagem em peso vivo:volume de solução, ocasionando redução da qualidade da água durante o tratamento de imersão. Este problema pode ser resolvido em futuros trabalhos, a partir do estudo das densidades de estocagem durante os banhos.

A partir destes resultados, novos trabalhos devem ser realizados para o ajuste de outros parâmetros (Piferrer, 2001), como dose, duração dos tratamentos, densidade de estocagem, manejo, período de imersão e outros, a fim de aprimorar a técnica de reversão sexual por meio de banhos de imersão e viabilizar sua aplicação efetiva em protocolos comerciais de reversão sexual, melhorando a eficiência dos tratamentos.

\section{Conclusões}

Os tratamentos hormonais em diferentes idades, por meio de banhos de imersão em solução contendo $17 \alpha$-metiltestosterona, não influenciaram os índices de desempenho zootécnico. O período ontogênico de maior sensibilidade de tilápias-do-nilo (Oreochromis niloticus) aos tratamentos foi aos 23,3 dias pós-eclosão.

\section{Literatura Citada}

ALCESTE, C.; JORRY, D.E. Análisis de las tendencias actuales en la comercialización de tilapia en los Estados Unidos de Norteamérica y la Unión Europea. In: AQUICULTURA BRASIL, 10., 1998, Recife. Anais... Recife: Associação Brasileira de Aquicultura, 1998. p.349-364.

ALVENIDA-CASAUAY, A.; CARIÑO, V.S. Gonadal sex differentiation in Oreochromis niloticus. In: INTERNATIONAL SYMPOSIUM ON TILAPIA AQUACULTURE, 2., 1988, Manila. Proceedings... Manila: International Center for Living Aquatic Resources Management, 1988. p.121-124.

APPLE, H.B.; LEBOUTE, E.M. Masculinização de pós-larvas de tilápia do Nilo (Oreochromis niloticus) utilizando andrógenos através de tratamentos de imersão. In: ENCONTRO SUL BRASILEIRO DE AQUICULTURA, 3., 1995, Ibirubá. Anais... Ibirubá: Cooperativa Agrícola Mista General Osório Ltda./ Universidade Federal do Rio Grande do Sul, 1995. p.113-119.

BEARDMORE, J.A.; MAIR, G.C.; LEWIS, R.I. Monosex male production in finfish as exemplified by tilapia: applications, problems, and prospects. Aquaculture, v.197, p.283-301, 2001 .

BLÁZQUEZ, M.; PIFERRER, F.; ZANUY, S. et al. Development of sex control techniques for European sea bass (Diacentrarchus labrax L.) aquaculture: effects of dietary $17 \alpha$-metiltestosterone prior to sex differentiation. Aquaculture, v.135, p.329-342, 1995.

BLÁZQUEZ, M.; ZANUY, S.; CARRILlo, M. et al. Critical period of androgen - inducible sex differentiation in a teleost fish, the European sea bass. Journal of Fish Biology, v.58, p.342358, 2001.

BOMBARDELLI, R.A.; HAYASHI, C. Feminilização de larvas de tilápia do Nilo (Oreochromis niloticus L.) a partir de banhos de imersão com valerato-de-estradiol. Revista Brasileira de Zootecnia, v.34, n.2, p.357-364, 2005b

BOMBARDELLI, R.A.; HAYASHI, C. Masculinização de larvas de tilápia do Nilo (Oreochromis niloticus L.) a partir de banhos de imersão com $17 \alpha$-metiltestosterona. Revista Brasileira de Zootecnia, v.34, n.2, p.365-372, 2005a

BOMBARDELLI, R.A.; HAYASHI, C.; MEURER, F. et al. Masculinização de larvas de tilápia do Nilo (Oreochromis niloticus) por banhos de imersão em $17 \alpha$-metiltestosterona. Varia Scientia, v.3, n.6, p.83-93, 2003.

BORGHETTI, N.R.B.; OSTRENSKY, A.; BORGHETTI, J.R. Aqüicultura - Uma visão geral sobre a produção de organismos aquáticos no Brasil e no mundo. Curitiba: Grupo Integrado de Aqüicultura e Estudos Ambientais, 2003. 128p.

BOSCOLO, W.R.; HAYASHI, C.; MEURER, F. Digestibilidade aparente da energia e nutrientes de alimentos convencionais e alternativos para a tilápia do Nilo (Oreochromis niloticus). Revista Brasileira de Zootecnia, v.31, n.2, p.539-545, 2002.

BOSCOLO, W.R.; HAYASHI, C.; SOARES, C.M. et al. Desempenho e características de carcaça de machos revertidos de tilápias do Nilo (Oreochromis niloticus), linhagens tailandesa e comum, nas fases inicial e de crescimento. Revista Brasileira de Zootecnia, v.30, n.5, p.1391-1396, 2001

BOYD, C.E. Water quality in ponds for aquaculture. Alabama: Birmingham Publishing Co, 1990. 482p.

CAMPOS-RAMOS, R.; HARVEY, S.C.; MCANDREW, B.J. et al An investigation of sex determination in the Mozambique tilapia, Oreochromis mossambicus, using synaptonemal complex analysis, fish, sex reversal and gynogenesis. Aquaculture, v.221, p.125-140, 2003.

DESPREZ, D.; GÉRAZ, E.; HOAREAU, M.C. et al. Production of a high percentage of male offspring with a natural androgen, $11 \beta$-hydroxyandrostenedione (11bOHA4), in Florida red tilapia. Aquaculture, v.216, p.55-65, 2003. 
ECKSTEIN, B.; SPIRA, M. Effects of sex hormones on gonadal differentiation in a cichlid, Tilapia aurea. Biological Bulletin, v.7, p.482-489, 1965.

FIEST, G.; YEOH, C.; FITZPATRICK, M.S. et al. The production of functional sex - reversed male rainbow trout with $17 \alpha$-metiltestosterone and 11ß-hydroxyandrostenedione. Aquaculture, v.131, p.145-152, 1995.

GALE, W.L.; FITZPATRICK, M.S.; LUCERO, M. et al. Masculinization of Nile tilapia (Oreochromis niloticus) by immersion in androgens. Aquaculture, v.178, p.349-357, 1999.

GOETZ, F.W.; DONALDSON, E.M.; HUNTER, G.A. et al. Effects of estradiol $17 \beta$ and $17 \alpha$ methyltestosterone on gonadal differentiation in the coho salmon, Oncorhynchus kisutch. Aquaculture, v.17, p.267-278, 1979.

HAYASHI, C.; BOMBARDELLI, R.A.; MEURER, F. Masculinização de larvas de tilápia do Nilo (Oreochromis niloticus) por banhos de imersão e o andrógeno dissolvido em solução de dimetilsulfóxido (DMSO). Acta Scientiarum - Animal Sciences, v.26, n.2, p.209-215, 2004.

HAYASHI, C.; BOSCOLO, W.R.; SOARES, C.M. et al. Exigência de proteína digestível para larvas de tilápia do Nilo (Oreochromis niloticus) durante a reversão sexual. Revista Brasileira de Zootecnia, v.31, n.2, p.823-829, 2002.

HAYASHI, C.; BOSCOLO, W.R.; SOARES, C.M. et al. Uso de diferentes graus de moagem dos ingredientes em dietas para tilápia do Nilo (Oreochromis niloticus L.) na fase de crescimento. Acta Scientiarum - Animal Sciences, v.21, n.3, p.733-737, 1999.

HIOTT, A.E.; PHELPS, R.P. Effects of initial age and size on sex reversal of Oreochromis niloticus fry using methyltestosterone. Aquaculture, v.112, p.301-308, 1993.

KOGER, C.S.; TEH, S.J.; HINTON, D.E. Determining the sensitive developmental stages of intersex induction in medaka (Oryzias latipes) exposed to 17 beta - estradiol or testosterone. Marine Environmental Research, v.50, n.1-5, p.201-206, 2000.

KRISFALUSI, M.; NAGLER, J.J. Induction of gonadal intersex in genotypic male rainbow trout (Oncorhynchus mykiss) embryos following immersion in estradiol - 17 $\beta$. Molecular Reproduction and Development, v.56, n.4, p.495-501, 2000.

LEONHARDT, J.H. Efeito da reversão sexual em tilápia do Nilo, Oreochromis niloticus (Linnaeus, 1757). Jaboticabal: Universidade Estadual Paulista, 1997. 128p. Tese (Doutorado em Aquicultura) - Universidade Estadual Paulista, 1997.

MACINTOSH, D.J.; LITTLE, D.C. Nile tilapia (Oreochromis niloticus) In: BROMAGE, N.R.; ROBERTS, R.J. (Eds.) Broodstock management and egg and larval quality. London: Blackwell Science Ltda, 1995. p.277-320.

MEURER, F.; HAYASHI, C.; BOSCOLO, W.R. Digestibilidade aparente de alguns alimentos protéicos pela tilápia do Nilo (Oreochromis niloticus). Revista Brasileira de Zootecnia, v.32, n.8, p.1801-1809, 2003b.

MEURER, F.; HAYASHI, C.; BOSCOLO, W.R. Influência do processamento da ração no desempenho e sobrevivência da tilápia do Nilo durante a reversão sexual. Revista Brasileira de Zootecnia, v.32, n.2, p.262-267, 2003a.

MEURER, F.; HAYASHI, C.; SOARES, C.M. et al. Utilização de levedura "spray dried" na alimentação de tilápia do Nilo (Oreochromis niloticus L.). Acta Scientiarum - Animal Sciences, v.22, n.4, p.479-484, 2000.

PANDIAN, T.J.; SHEELA, S.G. Hormonal induction of sex reversal in fish. Aquaculture, v.138, p.1-22, 1995
PHELPS, R.P.; POPMA, T.J. Sex reversal of tilapia In: COSTAPIERCE, B.A.; RAKOCY, J.E (Eds.) Tilapia aquaculture in the Americas. Louisiana: World Aquaculture Society, 2000. v.2, p.34-59.

PIFERRER, F. Endocrine sex control strategies for the feminization of teleost fish. Aquaculture, v.197, p.229-281, 2001

PIFERRER, F.; DONALDSON, E.M. Gonadal differentiation in coho salmon, Oncorhynchus kisutch, after a single treatment with androgen or estrogen at different stages during ontogenesis. Aquaculture, v.77, p.251-262, 1989.

POPMA, T.J.; PHELPS, R.P. Status report to commercial tilápia producers on monosex fingerling productions techniques. In: AQUiCultura Brasil, 10., 1998, Recife. Anais... Recife: Associação Brasileira de Aqüicultura, 1998. p.127-145.

POPMA, T.J.; GREEN, B.W. Aquacultural production manual: sex reversal of tilapia in earthen ponds. Research and Development Series, v.35, p.1-15, 1990.

SANCHES, L.E.F.; HAYASHI, C. Effect of feeding frequency on Nile tilapia, Oreochromis niloticus (L.) fries performance during sex reversal in hapas. Acta Scientiarum - Animal Sciences, v.23, n.4, p.871-876, 2001.

SIPAÚBA-TAVARES, L.H.S. Limnologia aplicada à aquicultura. Jaboticabal: FINEP, 1995. 70p.

SPECKER, J.L.; CHANDLEE, M.K. Methodology for estradiol treatment in marine larval and juvenile fish: uptake and clearance in summer flounder. Aquaculture, v.217, p.663$672,2003$.

STICKNEY, R.R. Status of research on tilápia In: COSTA-PIERCE, B.A.; RAKOCY, J.E. (Eds.). Tilapia aquaculture in the America. Louisiana: World Aquaculture Society, 2000. v.2, p. 21-33

STRUSSMANN, C.A.; TAKASHIMA, F.; TODA, K. Sex differentiation and hormonal feminization in pejerrey Odontesthes bonariensis. Aquaculture, v.139, p.31-45, 1996.

TORRANS, L.; MERIWETHER, F.; LOWELL, F. Sex - reversal of Oreochromis aureus by immersion in Mibolerone, a synthetic steroid. Journal of the World Aquaculture Society, v.19, n.3, p.97-102, 1988.

TURNER, G.F.; ROBINSON, R.L. Reproductive biology, mating systems and parental care In: BEVERIDGE, M.C.M.; MCANDREW, B.J. (Eds.) Tilapias: biology and explotation. London: Kluwer Academic Publishers, 2000. p.33-58.

UNIVERSIDADE FEDERAL DE VIÇOSA - UFV. SAEG - Sistema para Análises Estatísticas e Genéticas (Manual do usuário). Versão, 7.1. Viçosa, MG: 1997. 150p.

WASSERMANN, G.J.; AFONSO, L.O.B. Sex reversal in Nile tilapia (Oreochromis niloticus) by androgen immersion. Aquaculture Research, v.34, p.65-71, 2003.

WASSERMANN, G.J.; AFONSO, L.O.B.; OTT, R.P. et al. Masculinização de larvas de tilápia do Nilo (Oreochromis niloticus), usando andrógenos, através de banhos de imersão. In: REUNIÃO ANUAL DA SOCIEDADE BRASILEIRA DE Zootecnia, 37., 2000, Viçosa, MG. Anais... Viçosa, MG: Sociedade Brasileira de Zootecnia, 2000. (CD-ROM).

YAMAMOTO, T. Sex differentiation In: HOAR, W.S.; RANDALL D.J. (Eds.) Fish physiology. New York: Academic Press, 1969. v.3, p.117-75.

Recebido: $12 / 12 / 05$ Aprovado: 14/08/06 